\title{
On the existence of three solutions of Dirichlet fractional systems involving the $p$-Laplacian with Lipschitz nonlinearity
}

\author{
Rafik Guefaifia ${ }^{1}$, Salah Boulaaras ${ }^{2,3 *^{*}}$ and Fares Kamache ${ }^{1}$
}

On the occasion of the 80th birthday of the second author's mother, Mrs. Fatma Bint Al-Tayeb Zeghdoud.

\author{
"Correspondence: \\ saleh_boulaares@yahoo.fr; \\ s.Boularas@qu.edu.sa \\ 2Department of Mathematics, \\ College of Sciences and Arts, \\ Qassim University, Al-Rass, Kingdom \\ of Saudi Arabia \\ ${ }^{3}$ Laboratory of Fundamental and \\ Applied Mathematics of Oran \\ (LMFAO), University of Oran 1, \\ Ahmed Benbella, Algeria \\ Full list of author information is \\ available at the end of the article
}

\begin{abstract}
A class of perturbed fractional nonlinear systems is studied. The dynamical system possesses two control parameters and a Lipschitz nonlinearity order of $p-1$. The multiplicity of the weak solutions is proved by means of the variational method and by Ricceri critical points theorems. An illustrative example is analyzed in order to highlight the obtained result.
\end{abstract}

MSC: 35J60; 35B30; 35B40; 35J15; 35J25

Keywords: Fractional differential equations; Riemann-Liouville fractional derivatives; Variational methods; Three solutions; $p$-Laplacian

\section{Springer}

\section{Introduction}

Fractional differential equations have proved to be promising tools in the modeling of diverse phenomena in various fields, such as physics, chemistry, biology, engineering and economics. In recent years, there was a significant development in fractional differential equations due to the possibility of accounting for a larger class of memory properties. For instance, consider the studies of Miller and Ross [1], Boulaaras et al. [2, 3], Podlubny [4], Hilfer [5], Kilbas et al. [6], and the related papers [1, 7-16] and the references therein.

Critical point theory was very useful in determining the existence of solutions to complete differential equations with certain boundary conditions; see, for example, in the extensive literature on the subject, the classical books [17-19], and the references therein. However, so far, some problems were created for fractional boundary value problems (F-BVP) by exploiting this approach, where it is often very difficult to create suitable spaces and functions for fractional problems.

C) The Author(s) 2020. This article is licensed under a Creative Commons Attribution 4.0 International License, which permits use, sharing, adaptation, distribution and reproduction in any medium or format, as long as you give appropriate credit to the original author(s) and the source, provide a link to the Creative Commons licence, and indicate if changes were made. The images or other third party material in this article are included in the article's Creative Commons licence, unless indicated otherwise in a credit line to the material. If material is not included in the article's Creative Commons licence and your intended use is not permitted by statutory regulation or exceeds the permitted use, you will need to obtain permission directly from the copyright holder. To view a copy of this licence, visit http://creativecommons.org/licenses/by/4.0/. 
In [20], the authors investigated the following nonlinear fractional differential equation depending on two parameters:

$$
\left\{\begin{aligned}
{ }_{t} D_{T}^{\alpha_{i}}\left(a_{i}(t){ }_{0} D_{t}^{\alpha_{i}} u_{i}(t)\right) \\
\quad=\lambda F_{u_{i}}\left(t, u_{1}(t), u_{2}(t), \ldots, u_{n}(t)\right) \\
\quad+\mu G_{u_{i}}\left(t, u_{1}(t), u_{2}(t), \ldots, u_{n}(t)\right)+h_{i}\left(u_{i}\right) \quad \text { a.e }[0, T], \\
u_{i}(0)=u_{i}(T)=0,
\end{aligned}\right.
$$

for $1 \leq i \leq n$, where $\alpha_{i} \in(0 ; 1],{ }_{0} D_{t}^{\alpha_{i}}$ and ${ }_{t} D_{T}^{\alpha_{i}}$ are the left and right Riemann-Liouville fractional derivatives of order $\alpha_{i}$, respectively, with $a_{i} \in L^{\infty}([0, T])$ for

$$
a_{i 0}=\operatorname{ess} \inf _{[0, T]} a_{i}(t)>0 \quad \text { for } 1 \leq i \leq n, \lambda, \mu
$$

are positive parameters, $F, G:[0, T] \times \mathbb{R}^{n} \longrightarrow \mathbb{R}$ are measurable functions with respect to $t \in[0, T]$ for every $\left(x_{1}, \ldots, x_{n}\right) \in \mathbb{R}^{n}$ and are $C^{1}$ with respect to $\left(x_{1}, \ldots, x_{n}\right) \in \mathbb{R}^{n}$ for a.e. $t \in$ $[0, T], F_{u_{i}}$ and $G_{u_{i}}$ denote the partial derivative of $F$ and $G$ with respect to $u_{i}$, respectively, and $h_{i}: \mathbb{R} \rightarrow \mathbb{R}$ are Lipschitz continuous functions with the Lipschitz constants $L_{i}>0$ for $1 \leq i \leq n$, i.e.,

$$
\left|h_{i}\left(x_{1}\right)-h_{i}\left(x_{2}\right)\right| \leq L_{i}\left|x_{1}-x_{2}\right|
$$

for every $x_{1}, x_{2} \in \mathbb{R}$, and $h_{i}(0)=0$ for $1 \leq i \leq n$. Motivated by [21] and [22], using a three critical points theorem obtained in [23], which is recalled in the next section (Theorem 2), the existence of at least three solutions for this system is demonstrated.

For example, according to some assumptions, in [24], by using variational methods the authors obtained the existence of at least one weak solution for the following $p$-Laplacian fractional differential equation [24]:

$$
\left\{\begin{array}{l}
{ }_{t} D_{T}^{\alpha}\left(\phi_{p}\left({ }_{0} D_{t}^{\alpha} u(t)\right)\right)=\lambda f(t, u(t)) \quad \text { a.e. } t \in[0, T] \\
u(0)=u(T)=0
\end{array}\right.
$$

where ${ }_{0} D_{t}^{\alpha}$ and ${ }_{t} D_{T}^{\alpha}$ are the left and right Riemann-Liouville fractional derivatives with $0<\alpha \leq 1$, respectively, the function $\phi_{p}(s)=|s|^{p-2} s, p>1$. Taking a class of fractional differential equation with $p$-Laplacian operator as a model, Li et al. investigated the following equation recently [25]:

$$
\left\{\begin{array}{l}
{ }_{t} D_{T}^{\alpha}\left(\frac{1}{w(t)^{p-2}} \varphi_{p}\left({ }_{0} D_{t}^{\alpha} u(t)\right)\right)+\lambda u(t)=f\left(t, u,{ }_{0}^{c} D_{t}^{\alpha} u(t)\right)+h(u(t)) \quad \text { a.e. } t \in[0, T] \\
u(0)=u(T)=0
\end{array}\right.
$$

with $\frac{1}{p}<\alpha \leq 1, \lambda$ a non-negative real parameter.

The function

$$
\varphi_{p}(s)=|s|^{p-2} s, \quad p \geq 2, f:[0 ; T] \times \mathbb{R} \times \mathbb{R} \rightarrow \mathbb{R},
$$

is continuous and $h: \mathbb{R} \rightarrow \mathbb{R}$ is a Lipschitz continuous function. 
By using the mountain pass theorem combined with iterative technique, the authors obtained the existence of at least one solution for problem (1.3). In addition, in [20], three weak solutions for a new class of fractional $p$-Laplacian for boundary value Systems were established by using variational methods and critical point theory. In contrast, motivated by [21] and [22], the existence of at least three solutions for system (1.4) is demonstrated in the present paper, by means of the three critical points theorem obtained in [23], which is recalled in the next section (Theorem 2). This theorem has been successfully employed to establish the existence of at least three solutions for the case of perturbed boundary value problems; see [7, 26-28] and [29].

Consider the following fractional nonlinear system:

$$
\left\{\begin{aligned}
&{ }_{t} D_{T}^{\alpha_{i}} \phi_{p}\left({ }_{0} D_{t}^{\alpha_{i}} u_{i}(t)\right) \\
&=\lambda \lambda F_{u_{i}}\left(t, u_{1}(t), u_{2}(t), \ldots, u_{n}(t)\right) \\
&+\mu G_{u_{i}}\left(t, u_{1}(t), u_{2}(t), \ldots, u_{n}(t)\right) \\
&+h_{i}\left(u_{i}(t)\right) \quad \text { a.e. } t \in[0, T], \text { for } 1 \leq i \leq n \\
& u_{i}(0)=u_{i}(T)=0,
\end{aligned}\right.
$$

where $\alpha_{i} \in(0 ; 1], \phi_{p}(t)=|t|^{p-2} t, t \neq 0, \phi_{p}(0)=0, p>1,{ }_{0} D_{t}^{\alpha_{i}}$ and ${ }_{t} D_{T}^{\alpha_{i}}$ are the left and right Riemann-Liouville fractional derivatives of order $\alpha_{i}$, respectively, for $1 \leq i \leq n, \lambda$ and $\mu$ are positive parameters, and $F, G:[0, T] \times \mathbb{R}^{n} \rightarrow \mathbb{R}$ are measurable functions with respect to $t \in[0, T]$ for every $\left(x_{1}, x_{2}, \ldots, x_{n}\right) \in \mathbb{R}^{n}$ and are $C^{1}$ with respect to $\left(x_{1}, x_{2}, \ldots, x_{n}\right) \in \mathbb{R}^{n}$.for a.e. $t \in[0, T], F_{u_{i}}, G_{u_{i}}$ denote the partial derivative of $F$ and $G$ with respect to $u_{i}$, respectively, and $h_{i}: \mathbb{R} \rightarrow \mathbb{R}$ are Lipschitz continuous functions of order $(p-1)$ with Lipschizian constants $L_{i}>0$ for $1 \leq i \leq n$, i.e.,

$$
\left|h_{i}\left(x_{1}\right)-h_{i}\left(x_{2}\right)\right| \leq L_{i}\left|x_{1}-x_{2}\right|^{p-1}
$$

for every $x_{1}, x_{2} \in \mathbb{R}$, and $h_{i}(0)=0$ for $1 \leq i \leq n$.

In this paper, the following conditions are assumed:

$\left(H_{0}\right) \alpha_{i} \in\left(\frac{1}{p} ; 1\right]$ for $1 \leq i \leq n$.

(F1) for every $M>0$ and every $1 \leq i \leq n$,

$$
\sup _{\left|\left(x_{1}, x_{2}, \ldots, x_{n}\right)\right| \leq M}\left|F_{u_{i}}\left(t, x_{1}, x_{2}, \ldots, x_{n}\right)\right| \in L^{1}([0, T]) .
$$

(F2) $F(t, 0,0, \ldots, 0)=0$ for a.e. $t \in[0, T]$.

(G) for every $M>0$ and every $1 \leq i \leq n$,

$$
\sup _{\left|\left(x_{1}, x_{2}, \ldots, x_{n}\right)\right| \leq M}\left|G_{u_{i}}\left(t, x_{1}, x_{2}, \ldots, x_{n}\right)\right| \in L^{1}([0, T]) .
$$

This rest of this paper is organized as follows. The next section presents the necessary preliminary to develop the main contribution of this paper. In Sect. 3, the main result (Theorem 2) is derived, and meaningful consequences (Corollaries 1 and 2 and Example 1) are presented. 


\section{Preliminaries}

For the sake of clarity, the necessary definitions and properties of fractional calculus are presented below.

Definition 1 ([6]) Let $u$ be a function defined on $[a, b]$. The left and right RiemannLiouville fractional derivatives of order $\alpha>0$ for a function $u$ are defined by

$$
{ }_{a} D_{t}^{\alpha} u(t):=\frac{d^{n}}{d t^{n}}{ }_{a} D_{t}^{\alpha-n} u(t)=\frac{1}{\Gamma(n-\alpha)} \frac{d^{n}}{d t^{n}} \int_{a}^{t}(t-s)^{n-\alpha-1} u(s) d s
$$

and

$$
{ }_{t} D_{b}^{\alpha} u(t):=(-1)^{n} \frac{d^{n}}{d t^{n}}{ }_{t} D_{b}^{\alpha-n} u(t)=\frac{(-1)^{n}}{\Gamma(n-\alpha)} \frac{d^{n}}{d t^{n}} \int_{t}^{b}(t-s)^{n-\alpha-1} u(s) d s,
$$

for every $t \in[a, b]$, provided the right-hand sides are pointwise defined on $[a, b]$, where $n-1 \leq \alpha<n$ and $n \in \mathbb{N}$.

Here, $\Gamma(\alpha)$ is the gamma function, given by

$$
\Gamma(\alpha):=\int_{0}^{+\infty} z^{\alpha-1} e^{-z} d z
$$

The set $A C^{n}([a, b], \mathbb{R})$ corresponds to the space of functions $u:[a, b] \rightarrow \mathbb{R}$ such that $u \in C^{n-1}([a, b], \mathbb{R})$ and $u^{(n-1)} \in A C^{n}([a, b], \mathbb{R})$. Here, as usual, $C^{n-1}([a, b], \mathbb{R})$ denotes the set of mappings that are $(n-1)$ times continuously differentiable on $[a, b]$. In particular, $A C([a, b], \mathbb{R}):=A C^{1}([a, b], \mathbb{R})$.

Proposition 1 ([30]) The following property of fractional integration holds:

$$
\int_{a}^{b}\left[{ }_{a} D_{t}^{-\alpha} u(t)\right] v(t) d t=\int_{a}^{b}\left[{ }_{t} D_{b}^{-\alpha} v(t)\right] u(t) d t, \quad \alpha>0,
$$

provided that $u \in L^{p}([a, b], \mathbb{R}), v \in L^{q}([a, b], \mathbb{R})$ and $p \geq 1, q \geq 1, \frac{1}{p}+\frac{1}{q} \leq 1+\alpha$ or $p \neq 1$, $q \neq 1, \frac{1}{p}+\frac{1}{q}=1+\alpha$.

Proposition 2 ([31]) If $u(a)=u(b)=0, u \in L^{\infty}\left([a, b], \mathbb{R}^{N}\right), v \in L^{1}([a, b], \mathbb{R})$, or $v(a)=v(b)=$ $0, v \in L^{\infty}\left([a, b], \mathbb{R}^{N}\right), u \in L^{1}([a, b], \mathbb{R})$, then

$$
\int_{a}^{b}\left[{ }_{a} D_{t}^{\alpha} u(t)\right] v(t) d t=\int_{a}^{b}\left[{ }_{t} D_{b}^{\alpha} v(t)\right] u(t) d t, \quad 0<\alpha \leq 1 .
$$

To establish a variational structure for the main problem, it is necessary to construct appropriate function spaces. Following [32], $C_{0}^{\infty}([0, T], \mathbb{R})$ denotes the set of all functions $g \in C^{\infty}([0, T], \mathbb{R})$ with $g(0)=g(T)=0$.

Definition 2 ([24] $)$ For $0<\alpha_{i} \leq 1$, and for $1 \leq i \leq n$, the fractional derivative space $E_{0}^{\alpha i, p}$ is defined by

$$
E_{0}^{\alpha i, p}=\left\{u \in L^{p}([0, T], \mathbb{R}):{ }_{0} D_{t}^{\alpha_{i}} u_{i} \in L^{p}, u(0)=u(T)=0\right\}
$$


with the norm

$$
\left\|u_{i}\right\|_{\alpha_{i}, p}=\left(\left\|u_{i}\right\|_{L^{p}}^{p}+\left\|_{0} D_{t}^{\alpha_{i}} u_{i}\right\|_{L^{p}}^{p}\right)^{\frac{1}{p}}, \quad \forall u_{i} \in E_{0}^{\alpha i, p}
$$

where

$$
\left\|u_{i}\right\|_{L^{p}}=\left(\int_{0}^{T}\left|u_{i}\right|^{2} d t\right)^{\frac{1}{p}}
$$

is the norm of $L^{p}([0, T], \mathbb{R})$ for $1 \leq i \leq n$, and

$$
\left\|u_{i}\right\|_{\infty}=\max _{t \in[0, T]}\left|u_{i}(t)\right|
$$

From [24, Lemma 3.1], one finds that, for $0<\alpha_{i} \leq 1$ and $1<p<+\infty$, the space $E_{0}^{\alpha_{i}, p}$ is a reflexive and separable Banach space.

Lemma 1 ([24]) Let $0<\alpha_{i} \leq 1$, for $1 \leq i \leq n$ and $1<p<+\infty$, for all $u_{i} \in E_{0}^{\alpha_{i}, p}$ one has

$$
\begin{aligned}
\left\|u_{i}\right\|_{L^{p}} & \leq \frac{T^{\alpha_{i}}}{\Gamma\left(\alpha_{i}+1\right)}\left\|_{0} D_{t}^{\alpha_{i}} u_{i}\right\|_{L^{p}}, \\
\left\|u_{i}\right\|_{\infty} & \leq \frac{T^{\alpha_{i}-\frac{1}{p}}}{\Gamma\left(\alpha_{i}\right)\left(\left(\alpha_{i}-1\right) q+1\right)^{\frac{1}{q}}}\left\|_{0} D_{t}^{\alpha_{i}} u_{i}\right\|_{L^{p}} .
\end{aligned}
$$

Hence, it is possible to consider $E_{0}^{\alpha_{i}, p}$ with respect to the norm

$$
\left\|u_{i}\right\|_{\alpha_{i}, p}=\left\|_{0} D_{t}^{\alpha_{i}} u_{i}\right\|_{L^{p}}=\left(\int_{0}^{T}\left|{ }_{0} D_{t}^{\alpha_{i}} u_{i}\right|^{p} d t\right)^{\frac{1}{p}}, \quad \forall u_{i} \in E_{0}^{\alpha_{i}, p}
$$

for $1 \leq i \leq n$, which is equivalent to (2.1).

Hereafter, let $X$ be the Cartesian product of the $n$ spaces $E_{0}^{\alpha_{i}, p}$, i.e., $X=E_{0}^{\alpha_{1}, p} \times E_{0}^{\alpha_{2}, p} \times$ $\cdots \times E_{0}^{\alpha_{n}, p}$ equipped with the norm

$$
\|u\|=\sum_{i=1}^{n}\left\|u_{i}\right\|_{\alpha_{i}, p}, \quad u=\left(u_{1}, u_{2}, \ldots, u_{n}\right)
$$

where $\left\|u_{i}\right\|_{\alpha_{i}, p}$ is defined in (2.5). It is evident that $X$ is compactly embedded in $C([0, T], \mathbb{R})^{n}$

Definition 3 A weak solution of system (1.4) consists of any function $u=\left(u_{1}, u_{2}, \ldots, u_{n}\right) \in$ $X$, such that, for all $v=\left(v_{1}, v_{2}, \ldots, v_{n}\right) \in X$, one finds that

$$
\begin{aligned}
& \int_{0}^{T} \sum_{i=1}^{n}\left|{ }_{0} D_{t}^{\alpha_{i}} u_{i}(t)\right|^{p-2}{ }_{0} D_{t}^{\alpha_{i}} u_{i}(t)_{0} D_{t}^{\alpha_{i}} v_{i}(t) d t \\
& =\lambda \int_{0}^{T} \sum_{i=1}^{n} F_{u_{i}}\left(t, u_{1}(t), u_{2}(t), \ldots, u_{n}(t)\right) v_{i}(t) d t \\
& \quad+\mu \int_{0}^{T} \sum_{i=1}^{n} G_{u_{i}}\left(t, u_{1}(t), u_{2}(t), \ldots, u_{n}(t)\right) v_{i}(t) d t+\int_{0}^{T} \sum_{i=1}^{n} h_{i}\left(u_{i}(t)\right) v_{i}(t) d t .
\end{aligned}
$$


Remember the following result of [14, Theorem 1], with easy manipulations that are provided in the sequel.

Theorem 1 (Ricceri [14]) Let $X$ be a reflexive real Banach space; $\Phi: X \rightarrow \mathbb{R}$ be a continuously Gâteaux differentiable and sequentially weakly lower semicontinuous functional whose Gâteaux derivative admits a continuous inverse on $X^{*}$, bounded on bounded subsets of $X, \Psi: X \rightarrow \mathbb{R}$ a continuously Gâteaux differentiable functional whose Gâteaux derivative is compact such that

$$
\Phi(0)=\Psi(0)=0
$$

Assume that there exists $r>0$ and $\bar{x} \in X$, with $r<\Phi(\bar{x})$, such that

$\left(a_{1}\right) \frac{\sup _{\Phi(x) \leq r} \Psi(x)}{r}<\frac{\Phi(\bar{x})}{\Psi(\bar{x})}$

$\left(a_{2}\right)$ for each $\left.\lambda \in \Lambda_{r} ;=\right] \frac{\Phi(\bar{x})}{\Psi(\bar{x})}, \frac{r}{\sup _{\Phi(x) \leq r} \Psi(x)}[$, the functional $\Phi-\lambda \Psi$ is coercive.

Then, for each compact interval $[a, b] \subseteq \Lambda_{r}$, there exists $\rho>0$ with the following property: for every $\lambda \in[a, b]$ and every $C^{1}$ functional $\digamma: X \rightarrow \mathbb{R}$ with compact derivative, there exists $\delta>0$ such that, for each $\mu \in[0, \delta]$, the equation

$$
\Phi^{\prime}(x)-\lambda \Psi^{\prime}(x)-\mu \digamma^{\prime}(x)=0
$$

has at least three solutions in $X$ whose norms are less than $\rho$.

\section{The main results}

In the present section, the existence of multiple solutions for system (1.1) is discussed. For any $\varsigma>0, K(\varsigma)$ denotes

$$
\left\{\left(x_{1}, x_{2}, \ldots, x_{n}\right) \in \mathbb{R}^{n}: \frac{1}{p} \sum_{i=1}^{n}\left|x_{i}\right|^{n} \leq \varsigma\right\} .
$$

This set is one of the cornerstones of the given hypotheses for appropriate choices of $\varsigma$. For $u=\left(u_{1}, u_{2}, \ldots, u_{n}\right) \in X$ one has

$$
\Upsilon(u):=\sum_{i=1}^{n} \Upsilon_{i}\left(u_{i}\right)
$$

where

$$
\Upsilon_{i}(x):=\int_{0}^{T} H_{i}(x(s)) d s \quad \text { and } \quad H_{i}(x):=\int_{0}^{x} h_{i}(z) d z, \quad 1 \leq i \leq n,
$$

for every $t \in[0, T]$ and $x \in \mathbb{R}$. Moreover, let

$$
\begin{aligned}
c & :=\max _{1 \leq i \leq n}\left\{\frac{T^{\alpha_{i}-\frac{1}{p}}}{\Gamma\left(\alpha_{i}\right)\left(\left(\alpha_{i}-1\right) q+1\right)^{\frac{1}{q}}},\right. \\
k & :=\min _{1 \leq i \leq n}\left\{1-\frac{L_{i} T^{\alpha_{i} p}}{\left(\Gamma\left(\alpha_{i}+1\right)\right)^{p}}\right\}, \\
\tau & :=\max _{1 \leq i \leq n}\left\{1+\frac{L_{i} T^{p \alpha_{i}}}{\left(\Gamma\left(\alpha_{i}+1\right)\right)^{p}}\right\} .
\end{aligned}
$$


Theorem 2 Suppose that $k>0$ and the conditions $(F 1),(F 2),(G)$ and $(H)$ are satisfied. Furthermore, assume that there exist a positive constant $r$ and a function $\omega=\left(\omega_{1}, \omega_{2}, \ldots, \omega_{n}\right) \in$ $X$ such that

(i)

$$
\frac{1}{p} \sum_{i=1}^{n}\left\|\omega_{i}\right\|_{\alpha_{i}}^{p}>\frac{r}{k}
$$

(ii)

$$
\frac{r \int_{0}^{T} F\left(t, \omega_{1}, \omega_{2}, \ldots, \omega_{n}\right) d t}{\frac{1}{p} \sum_{i=1}^{n}\left\|\omega_{i}\right\|_{\alpha_{i}}^{p}-\Upsilon\left(\omega_{1}, \omega_{2}, \ldots, \omega_{n}\right)}-\int_{0}^{T} \max _{\left(x_{1}, x_{2}, \ldots, x_{n}\right) \in K\left(\frac{c r}{k}\right)} F\left(t, x_{1}, x_{2}, \ldots, x_{n}\right) d t>0
$$

(iii)

$$
\lim _{\left(\left|x_{1}\right|,\left|x_{2}\right|, \ldots,\left|x_{n}\right|\right) \rightarrow(+\infty,+\infty, \ldots,+\infty)} \sup \frac{\sup _{t \in[0, T]} F\left(t, x_{1}, x_{2}, \ldots, x_{n}\right)}{\frac{1}{p} \sum_{i=1}^{n}\left|x_{i}\right|^{p}} \leq 0
$$

Then, setting

$$
\left.\Lambda_{r}:=\right] \frac{\frac{1}{p} \sum_{i=1}^{n}\left\|\omega_{i}\right\|_{\alpha_{i}}^{p}-\Upsilon\left(\omega_{1}, \omega_{2}, \ldots, \omega_{n}\right)}{\int_{0}^{T} F\left(t, \omega_{1}, \omega_{2}, \ldots, \omega_{n}\right) d t}, \frac{r}{\int_{0}^{T} \max _{\left(x_{1}, x_{2}, \ldots, x_{n}\right) \in K\left(\frac{c r}{k}\right)} F\left(t, x_{1}, x_{2}, \ldots, x_{n}\right) d t}[\text {, }
$$

for each compact interval $[a, b] \subseteq \Lambda_{r}$, there exists $\rho>0$ with the following property: for every $\lambda \in[a, b]$ there exists $\delta>0$ such that, for each $\mu \in[0, \delta]$, system (1.4) admits at least three solutions in $X$ whose norms are less than $\rho$.

Proof For each $u=\left(u_{1}, u_{2}, \ldots, u_{n}\right) \in X$, define $\Phi, \Psi: X \rightarrow \mathbb{R}$ as

$$
\Phi(u):=\frac{1}{p} \sum_{i=1}^{n}\left\|u_{i}\right\|_{\alpha_{i}, p}^{p}-\Upsilon(u)
$$

and

$$
\Psi(u):=\int_{0}^{T} F\left(t, u_{1}(t), u_{2}(t), \ldots, u_{n}(t)\right) d t
$$

Clearly, $\Phi$ and $\Psi$ are continuously Gâteaux differentiable functionals whose Gâteaux derivatives at the point $u \in X$ are given by

$$
\begin{aligned}
& \Phi^{\prime}(u)(v):=\int_{0}^{T} \sum_{i=1}^{n}\left|{ }_{0} D_{t}^{\alpha_{i}} u_{i}(t)\right|^{p-2}{ }_{0} D_{t}^{\alpha_{i}} u_{i}(t){ }_{0} D_{t}^{\alpha_{i}} v_{i}(t) d t-\int_{0}^{T} \sum_{i=1}^{n} h_{i}\left(u_{i}(t)\right) v_{i}(t) d t \\
& \Psi^{\prime}(u)(v)=\int_{0}^{T} \sum_{i=1}^{n} F_{u_{i}}\left(t, u_{1}(t), u_{2}(t), \ldots, u_{n}(t)\right) v_{i}(t) d t
\end{aligned}
$$

for every $v=\left(v_{1}, v_{2}, \ldots, v_{n}\right) \in X$. Hence, $\Phi-\lambda \Psi \in C^{1}(X, \mathbb{R})$. Moreover, $\Psi^{\prime}: X \rightarrow X^{*}$ is a compact operator (see the proof of [21, Theorem 3.1]). Furthermore, similar to the proof 
of [22, Theorem 3.1], we can show that $\Phi$ is sequentially weakly lower semicontinuous. As concerns functional $\Phi$, it is easy to show that $\Phi$ is bounded on each bounded subset of $X$ and its derivative admits a continuous inverse on $X^{*}$. Moreover, we have $\Phi(0)=\Psi(0)=0$.

It is shown that the required hypothesis $\Phi(\bar{x})>r$ follows from $(i)$ and the definition of $\Phi$, by choosing $\bar{x}=\omega$. Indeed, since (1.5) holds for every $x_{1}, x_{2} \in \mathbb{R}$ and $h_{1}(0)=h_{2}(0)=\cdots=$ $h_{n}(0)=0$, one has $\left|h_{i}(x)\right| \leq L_{i}|x|^{p-1}, 1 \leq i \leq n$, for all $x \in \mathbb{R}$. Besides, it follows from (2.3) that

$$
\begin{aligned}
\Phi(\omega) & \geq \frac{1}{p} \sum_{i=1}^{n}\left\|\omega_{i}\right\|_{\alpha_{i}, p}^{p}-\left|\int_{0}^{T} \sum_{i=1}^{n} H_{i}\left(\omega_{i}(t)\right) d t\right| \\
& \geq \frac{1}{p} \sum_{i=1}^{n}\left\|\omega_{i}\right\|_{\alpha_{i}, p}^{p}-\frac{1}{p} \sum_{i=1}^{n} L_{i} \int_{0}^{T}\left|\omega_{i}\right|^{p_{i}} d t \\
& \geq \sum_{i=1}^{n}\left(\frac{1}{p}-\frac{L_{i} T^{\alpha_{i} p}}{p\left(\Gamma\left(\alpha_{i}+1\right)\right)^{p_{i}}}\right)\left\|\omega_{i}\right\|_{\alpha_{i}, p}^{p} \\
& \geq \frac{k}{p} \sum_{i=1}^{n}\left\|\omega_{i}\right\|_{\alpha_{i}, p}^{p}>r .
\end{aligned}
$$

From (2.2) and (2.4), for every $u_{i} \in E_{0}^{\alpha_{i}, p}$ one has

$$
\max _{t \in[0, T]}\left|u_{i}(t)\right|^{p} \leq c\left\|u_{i}\right\|_{\alpha_{i}, p}^{p}
$$

for each $u=\left(u_{1}, u_{2}, \ldots, u_{n}\right) \in X$. From (2.4), (3.1) and (3.2), for each $r>0$ one obtains

$$
\begin{aligned}
\Phi^{-1} & ((-\infty ; r]) \\
& =\left\{u=\left(u_{1}, u_{2}, \ldots, u_{n}\right) \in X: \Phi(u) \leq r\right\} \\
& \subseteq\left\{u=\left(u_{1}, u_{2}, \ldots, u_{n}\right) \in X: \frac{1}{p} \sum_{i=1}^{n}\left\|u_{i}\right\|_{\alpha_{i}, p}^{p} \leq \frac{r}{k}\right\} \\
& \subseteq\left\{u=\left(u_{1}, u_{2}, \ldots, u_{n}\right) \in X: \frac{1}{p} \sum_{i=1}^{n} \frac{\left(\Gamma\left(\alpha_{i}\right)\right)^{p}\left(\left(\left(\alpha_{i}-1\right) q+1\right)\right)^{\frac{p}{q}}}{T^{\alpha_{i} p-1}}\left\|u_{i}\right\|_{\infty}^{p} \leq \frac{r}{k}\right\} \\
& \subseteq\left\{u=\left(u_{1}, u_{2}, \ldots, u_{n}\right) \in X: \frac{1}{p} \sum_{i=1}^{n}\left|u_{i}\right|^{p} \leq \frac{c r}{k}, \text { for all } t \in[0, T]\right\} .
\end{aligned}
$$

Then

$$
\begin{aligned}
\sup _{u \in \Phi^{-1}((-\infty ; r])} \Psi(u) & =\sup _{u \in \Phi^{-1}((-\infty ; r])} \int_{0}^{T} F\left(t, u_{1}(t), u_{2}(t), \ldots, u_{n}(t)\right) d t \\
& \leq \int_{0}^{T} \max _{\left(x_{1}, x_{2}, \ldots, x_{n}\right) \in K\left(\frac{c r}{k}\right)} F\left(t, x_{1}, x_{2}, \ldots, x_{n}\right) d t .
\end{aligned}
$$


Therefore, from the condition (ii), one gets

$$
\begin{aligned}
\sup _{u \in \Phi^{-1}((-\infty ; r])} \Psi(u) & \leq \int_{0}^{T} \max _{\left(x_{1}, x_{2}, \ldots, x_{n}\right) \in K\left(\frac{c r}{k}\right)} F\left(t, x_{1}, x_{2}, \ldots, x_{n}\right) d t \\
& <\frac{r \int_{0}^{T} F\left(t, \omega_{1}, \omega_{2}, \ldots, \omega_{n}\right) d t}{\frac{1}{p} \sum_{i=1}^{n}\left\|\omega_{i}\right\|_{\alpha_{i}, p}^{p}-\Upsilon\left(\omega_{1}, \omega_{2}, \ldots, \omega_{n}\right)} \\
& =\frac{r \int_{0}^{T} F\left(t, \omega_{1}, \omega_{2}, \ldots, \omega_{n}\right) d t}{\frac{1}{p} \sum_{i=1}^{n}\left\|\omega_{i}\right\|_{\alpha_{i}, p}^{p}-\Upsilon\left(\omega_{1}, \omega_{2}, \ldots, \omega_{n}\right)} \\
& =r \frac{\Psi(w)}{\Phi(w)}
\end{aligned}
$$

from which assumption $\left(a_{1}\right)$ of Theorem 1 follows. Fix $0<\epsilon<\frac{1}{p T c \lambda}$; from (iii) there is a constant $\tau_{\epsilon}$ such that

$$
F\left(t, x_{1}, x_{2}, \ldots, x_{n}\right) \leq \epsilon \sum_{i=1}^{n}\left|x_{i}\right|^{p}+\tau_{\epsilon_{i}}
$$

for every $t \in[0, T]$ and for every $\left(x_{1}, x_{2}, \ldots, x_{n}\right) \in \mathbb{R}^{n}$. Taking (2.4) into account, from (3.3), it follows that, for each $u \in X$,

$$
\begin{aligned}
\Phi(u)-\lambda \Psi(u) & =\frac{1}{p} \sum_{i=1}^{n}\left\|u_{i}\right\|_{\alpha_{i}, p}^{p}-\lambda \int_{0}^{T} F\left(t, u_{1}, u_{2}, \ldots, u_{n}\right) d t \\
& \geq \frac{1}{p} \sum_{i=1}^{n}\left\|u_{i}\right\|_{\alpha_{i}, p}^{p}-T \lambda c \epsilon \sum_{i=1}^{n}\left\|u_{i}\right\|_{\alpha_{i}, p}^{p}-\lambda \tau_{\epsilon} \\
& \geq\left(\frac{1}{p}-T \lambda c \epsilon\right) \sum_{i=1}^{n}\left\|u_{i}\right\|_{\alpha_{i}, p}^{p}-\lambda \tau_{\epsilon},
\end{aligned}
$$

and thus

$$
\lim _{\|u\| \rightarrow+\infty}(\Phi(u)-\lambda \Psi(u))=+\infty
$$

which means the functional $\Phi(u)-\lambda \Psi(u)$ is coercive for every parameter $\lambda$, in particular, for every $\lambda \in \Lambda \subset] \frac{\Phi(\omega)}{\Psi(\omega)}, \frac{r}{\sup _{\Phi(u)<r} \Psi(u)}$ [. Then also condition $\left(a_{2}\right)$ holds.

In addition, since $G:[0, T] \times \mathbb{R}^{n} \rightarrow \mathbb{R}$ is a measurable function with respect to $t \in[0, T]$ for every $\left(x_{1}, x_{2}, \ldots, x_{n}\right) \in \mathbb{R}^{n}$ belonging to $C^{1}$ with respect to $\left(x_{1}, x_{2}, \ldots, x_{n}\right) \in \mathbb{R}^{n}$ for a.e. $t \in[0, T]$ satisfying condition $(\mathbf{G})$, the functional

$$
\digamma(u)=\int_{0}^{T} G\left(t, u_{1}(t), u_{2}(t), \ldots, u_{n}(t)\right) d t
$$

is well defined and continuously Gâteaux differentiable on $X$ with a compact derivative, and

$$
\digamma^{\prime}(u)=\int_{0}^{T} \sum_{i=1}^{n} G_{u_{i}}\left(t, u_{1}(t), u_{2}(t), \ldots, u_{n}(t)\right) v_{i}(t) d t
$$


for all $\left(u_{1}, u_{2}, \ldots, u_{n}\right),\left(v_{1}, v_{2}, \ldots, v_{n}\right) \in X$. Thus, all the hypotheses of Theorem 1 are satisfied. Also note that the solutions of the equation

$$
\Phi^{\prime}(x)-\lambda \Psi^{\prime}(x)-\mu \digamma^{\prime}(x)=0
$$

are exactly the solutions of (1.4) (see [21]). So, the conclusion follows from Theorem 1 .

Example 1 Consider the following fractional boundary value problem:

$$
\left\{\begin{array}{l}
{ }_{t} D_{T}^{0,75} \phi_{3}\left({ }_{0} D_{t}^{0,75} u_{1}(t)\right)=\lambda F_{u_{1}}\left(t, u_{1}(t), u_{2}(t)\right)+\mu G_{u_{1}}\left(t, u_{1}(t), u_{2}(t)\right)+h_{1}\left(u_{1}(t)\right) \\
\quad \text { a.e. } t \in[0, T], \\
{ }_{t} D_{T}^{0,6} \phi_{3}\left({ }_{0} D_{t}^{0,6} u_{2}(t)\right)=\lambda F_{u_{2}}\left(t, u_{1}(t), u_{2}(t)\right)+\mu G_{u_{1}}\left(t, u_{1}(t), u_{2}(t)\right)+h_{2}\left(u_{2}(t)\right) \\
\quad \text { a.e. } t \in[0, T] \\
u_{1}(0)=u_{2}(0)=u_{1}(1)=u_{2}(1)=0
\end{array}\right.
$$

where $\alpha_{1}=0.75, \alpha_{2}=0.6, p=3, T=1, h_{1}\left(u_{1}\right)=\left(\sin \left(\frac{u_{1}}{2}\right)\right)^{2}, h_{2}\left(u_{2}\right)=\left(\arctan \left(\frac{u_{2}}{3}\right)\right)^{2}$ and $G$ : $[0,1] \times \mathbb{R}^{2} \rightarrow \mathbb{R}$ is an arbitrary function which is measurable with to respect to $t \in[0,1]$ for every $\left(x_{1}, x_{2}\right) \in \mathbb{R}^{2}$ and is $C^{1}$ with respect to $\left(x_{1}, x_{2}\right) \in \mathbb{R}^{2}$ for a.e. $t \in[0,1]$, satisfying

$$
\sup _{\left|\left(x_{1}, x_{2}\right)\right| \leq M}\left|G_{u_{i}}\left(t, x_{1}, x_{2}\right)\right| \in L^{1}([0, T])
$$

for every $M>0$ and $i=1,2$. Moreover, for all $\left(t, x_{1}, x_{2}\right) \in[0,1] \times \mathbb{R}^{2}$, put $F\left(t, x_{1}, x_{2}\right)=(1+$ $\left.t^{2}\right) H\left(x_{1}, x_{2}\right)$, where

$$
H\left(x_{1}, x_{2}\right)= \begin{cases}\left(x_{1}^{3}+x_{2}^{3}\right)^{2}, & x_{1}^{3}+x_{2}^{3} \leq 1 \\ 2 \sqrt{x_{1}^{3}+x_{2}^{3}}-\left(x_{1}^{3}+x_{2}^{3}\right), & x_{1}^{3}+x_{2}^{3}>1\end{cases}
$$

Obviously, $F(t, 0,0)=0$ for all $t \in[0,1]$, and a direct calculation shows that

$$
c \approx 1.0727, \quad k \approx 0.3559
$$

By choosing, for instance,

$$
\omega_{1}(t)=\Gamma(1,25) t(1-t), \quad \omega_{2}(t)=\Gamma(1,4) t(1-t)
$$

and $r=\frac{1}{10^{3}}$ all assumptions of Theorem 2 are satisfied. In fact, $\omega_{i}(0)=\omega_{i}(1)=0, i=1,2$, and

$$
{ }_{0} D_{t}^{0,75} \omega_{1}(t)=t^{0,25}-\frac{2 \Gamma(1,25)}{\Gamma(2,25)} t^{1,25}, \quad{ }_{0} D_{t}^{0,6} \omega_{2}(t)=t^{0,4}-\frac{2 \Gamma(1,4)}{\Gamma(2,4)} t^{1,4}
$$

Then one has

$$
\left\|\omega_{1}\right\|_{0.75}^{3} \approx 0.0498, \quad\left\|\omega_{2}\right\|_{0.6}^{3} \approx 0.0233
$$


which implies that the condition (i) holds, and

$$
\begin{aligned}
\frac{\int_{0}^{1} \max _{\left(x_{1}, x_{2}\right) \in \pi\left(\frac{c r}{k}\right)} F\left(t, x_{1}, x_{2}\right) d t}{r} & =\frac{12 c^{2} r}{k^{2}} \approx 0.1090 \\
& <\frac{\int_{0}^{1} F\left(t, \omega_{1}, \omega_{2}\right) d t}{\frac{1}{3} \sum_{i=1}^{2}\left\|\omega_{i}\right\|_{\alpha_{i}}^{3}-\Upsilon\left(\omega_{1}, \omega_{2}\right)} \approx 0.5548
\end{aligned}
$$

and

$$
\lim _{\left(\left|x_{1}\right|,\left|x_{2}\right|\right) \rightarrow(+\infty,+\infty)} \sup \frac{\sup _{t \in[0,1]} F\left(t, u_{1}, u_{2}\right)}{\frac{1}{3} \sum_{i=1}^{2}\left|u_{i}\right|^{3}}=0
$$

Thus, conditions (ii) and (iii) are satisfied. Then, in view of Theorem 2 for each $\lambda \epsilon$ ]1.8025, 9.1743[, system (3.4) has at least three weak solutions in $X=E_{0}^{.0,75,3} \times E_{0}^{.0,6,3}$.

Next, it is desirable to give a verifiable consequence of Theorem 2 for a fixed text function $\omega$. For a given constant $\gamma \in\left(0, \frac{1}{2}\right)$ and for all $1 \leq i \leq n$, set

$$
\begin{aligned}
& C_{i}\left(\alpha_{i}, \gamma\right)= \frac{1}{p(\gamma T)^{p}}\left\{\int_{0}^{\gamma T} t^{p\left(1-\alpha_{i}\right)} d t+\int_{\gamma T}^{(1-\gamma) T}\left(t^{1-\alpha_{i}}-(t-\gamma T)^{1-\alpha_{i}}\right)^{p} d t\right. \\
&\left.+\int_{(1-\gamma) T}^{T}\left(t^{1-\alpha_{i}}-(t-\gamma T)^{1-\alpha_{i}}\right)-\left(1-((1-\gamma) T)^{1-\alpha_{i}}\right)^{p}\right\}, \\
& \triangle=\min _{1 \leq i \leq n}\left\{\sum_{i=1}^{n} C_{i}\left(\alpha_{i}, \gamma\right)\right\}, \\
& \triangle^{\prime}=\max \left\{\sum_{i=1}^{n} C_{i}\left(\alpha_{i}, \gamma\right)\right\} .
\end{aligned}
$$

Corollary 1 Let assumption (iii) in Theorem 2 hold. Assume that there exist positive constants $d$ and $\eta$ such that $\frac{d}{\Delta c k n} \geq \eta^{p}$, and also

(j) $F\left(t, x_{1}, x_{2}, \ldots, x_{n}\right) \geq 0$, for each $\left(t, x_{1}, x_{2}, \ldots, x_{n}\right) \in[0, T] \times[0 ;+\infty) \times \cdots \times[0 ;+\infty)$;

(ji) $\frac{\int_{0}^{T} \max _{\left(x_{1}, x_{2}, \ldots, x_{n}\right) \in K(d)} F\left(t, x_{1}, x_{2}, \ldots, x_{n}\right) d t}{k d}<\frac{\int_{\gamma T}^{(1-\gamma) T} F\left(t, \Gamma\left(2-\alpha_{1}\right) \eta \ldots, \Gamma\left(2-\alpha_{n}\right) \eta\right) d t}{n c \tau \Delta^{\prime} \eta^{p}}$.

Then, setting

$$
\begin{aligned}
\Lambda_{1}:= & \left(\frac{n \tau \Delta^{\prime} \eta^{p}}{\int_{\gamma T}^{(1-\gamma) T} F\left(t, \Gamma\left(2-\alpha_{1}\right) \eta, \ldots, \Gamma\left(2-\alpha_{n}\right) \eta\right) d t},\right. \\
& \left.\frac{k d}{\int_{0}^{T} \max _{\left(x_{1}, x_{2}, \ldots, x_{n}\right) \in K(d)} F\left(t, x_{1}, x_{2}, \ldots x_{n}\right) d t}\right)
\end{aligned}
$$

for each compact interval $[a, b] \subseteq \Lambda_{1}$, there exists $\rho>0$ with the following property: for every $\lambda \in] a, b$, there exists $\delta$ such that, for each $\mu \in[0, \delta]$, system (1.4) admits at least three solutions in $X$ whose norms are less than $\rho$. 
Proof For $\gamma \in\left(0, \frac{1}{2}\right)$ choose $\omega(t)=\left(\omega_{1}(t), \ldots, \omega_{n}(t)\right)$ for every $t \in[0, T]$ with

$$
\omega_{i}(t)= \begin{cases}\frac{\Gamma\left(2-\alpha_{i}\right) \eta}{\gamma T} t, & t \in[0 ; \gamma T) \\ \Gamma\left(2-\alpha_{i}\right) \eta, & t \in[\gamma T ;(1-\gamma) T] \\ \frac{\Gamma\left(2-\alpha_{i}\right) \eta}{\gamma T}(t-T), & t \in((1-\gamma) T ; T]\end{cases}
$$

for $1 \leq i \leq n$, Clearly $\omega_{i}(0)=\omega_{i}(T)=0$ and $\omega_{i} \in L^{2}([0, T], \mathbb{R})$ for $1 \leq i \leq n$,A direct calculation shows that

$$
{ }_{0} D_{t}^{\alpha_{i}} \omega_{i}(t)= \begin{cases}\frac{\eta}{\gamma T} t^{1-\alpha_{i}}, & t \in[0 ; \gamma T), \\ \frac{\eta}{\gamma T}\left(t^{1-\alpha_{i}}-(t-\gamma T)^{1-\alpha_{i}}\right), & t \in[\gamma T ;(1-\gamma) T], \\ \frac{\eta}{\gamma T}\left(t^{1-\alpha_{i}}-(t-\gamma T)^{1-\alpha_{i}}-(t-(1-\gamma) T)^{1-\alpha_{i}}\right), & t \in((1-\gamma) T ; T],\end{cases}
$$

for $1 \leq i \leq n$. Furthermore,

$$
\begin{aligned}
\int_{0}^{T}\left|{ }_{0} D_{t}^{\alpha_{i}} \omega_{i}(t)\right|^{p} d t= & \left(\frac{\eta}{\gamma T}\right)^{p}\left\{\int_{0}^{\gamma T} t^{\left(1-\alpha_{i}\right) p} d t+\int_{h T}^{(1-\gamma) T}\left(t^{1-\alpha_{i}}-(t-\gamma T)^{1-\alpha_{i}}\right)^{p} d t\right. \\
& \left.+\int_{(1-h) T}^{T}\left(t^{1-\alpha_{i}}-(t-\gamma T)^{1-\alpha_{i}}-(t-(1-\gamma) T)^{1-\alpha_{i}}\right)^{p} d t\right\} \\
= & p \eta^{p} C_{i}\left(\alpha_{i}, h\right),
\end{aligned}
$$

for $1 \leq i \leq n$. Thus, $\omega \in X$, and

$$
\left\|\omega_{i}\right\|_{\alpha_{i}, p}^{p}=p \eta^{p} C_{i}\left(\alpha_{i}, h\right)
$$

with $1 \leq i \leq n$. This and (3.1) imply that

$$
\begin{aligned}
\Phi(\omega)=\Phi\left(\omega_{1}, \ldots, \omega_{n}\right) & =\frac{1}{p} \sum_{i=1}^{n}\left\|\omega_{i}\right\|_{\alpha_{i}, p}^{p}-\Upsilon\left(\omega_{i}\right) \\
& \geq \frac{k}{p} \sum_{i=1}^{n}\left\|\omega_{i}\right\|_{\alpha_{i}, p}^{p} \\
& \geq k \eta^{p} \sum_{i=1}^{n} C_{i}\left(\alpha_{i}, h\right) \\
& \geq n k \triangle \eta^{p} .
\end{aligned}
$$

Similarly to (3.1) and (3.5) one has

$$
\Phi(\omega) \leq n \tau \triangle^{\prime} \eta^{p}
$$

Let $r=\frac{k d}{c}$. From $\frac{d}{\Delta c k n}<\eta^{p}$, it is found as a result that

$$
\frac{1}{p} \sum_{i=1}^{n}\left\|\omega_{i}\right\|_{\alpha_{i}, p}^{p} \geq \Phi(\omega) \geq n k \Delta \eta^{p} \geq n k \Delta \times \frac{d}{\Delta c k n}=\frac{r}{k}
$$


which is assumption (i) of Theorem 2.

On the other hand, by using assumption (j), one can infer

$$
\begin{aligned}
\Psi(\omega) & :=\int_{0}^{T} F\left(t, \omega_{1}(t), \omega_{2}(t), \ldots, \omega_{n}(t)\right) d t \\
& \geq \int_{\gamma T}^{(1-\gamma) T} F\left(t, \Gamma\left(2-\alpha_{1}\right) \eta, \Gamma\left(2-\alpha_{2}\right) \eta, \ldots, \Gamma\left(2-\alpha_{n}\right) \eta\right) d t .
\end{aligned}
$$

Moreover, by condition (jj) one gets

$$
\begin{aligned}
\frac{\int_{0}^{T} \max _{\left(x_{1}, x_{2}, \ldots, x_{n}\right) \in K\left(\frac{c r}{k}\right)} F\left(t, x_{1}, x_{2}, \ldots x_{n}\right) d t}{r} \\
\quad=\frac{c \int_{0}^{T} \max _{\left(x_{1}, x_{2}, \ldots, x_{n}\right) \in K(d)} F\left(t, x_{1}, x_{2}, \ldots x_{n}\right) d t}{k d} \\
\quad<\frac{\int_{\gamma T}^{(1-\gamma) T} F\left(t, \Gamma\left(2-\alpha_{1}\right) \eta, \ldots, \Gamma\left(2-\alpha_{n}\right) \eta\right) d t}{n \tau \Delta^{\prime} \eta^{p}} \\
\leq \frac{\int_{\gamma T}^{(1-\gamma) T} F\left(t, \Gamma\left(2-\alpha_{1}\right) \eta, \ldots, \Gamma\left(2-\alpha_{n}\right) \eta\right) d t}{\Phi(\omega)} \\
\leq \frac{p \int_{0}^{T} F\left(t, \omega_{1}, \omega_{2}, \ldots, \omega_{n}\right) d t}{\sum_{i=1}^{n}\left\|\omega_{i}\right\|_{\alpha_{i}, p}^{p}-p \Upsilon\left(\omega_{1}, \omega_{2}, \ldots, \omega_{n}\right)},
\end{aligned}
$$

which implies that (ii) is satisfied. Thus, all the assumptions of Theorem 2 are satisfied and the proof is complete.

Corollary 2 Let $F: \mathbb{R}^{n} \rightarrow \mathbb{R}^{n}$ be a $C^{1}$-function and $F(0, \ldots, 0)=0$. Assume that there exist positive constants $d$ and $\eta$ such that $\frac{d}{\Delta c k n}<\eta^{p}$, and also

(H) $F\left(x_{1}, \ldots, x_{n}\right) \geq 0$, for each $\left(x_{1}, \ldots, x_{n}\right) \in[0 ;+\infty) \times \cdots \times[0 ;+\infty)$;

$(\mathrm{HH}) \frac{\max _{\left(x_{1}, x_{2}, \ldots, x_{n}\right) \in K(d)} F\left(x_{1}, x_{2}, \ldots x_{n}\right)}{k d}<\frac{(1-2 \gamma) F\left(\Gamma\left(2-\alpha_{1}\right) \eta, \ldots, \Gamma\left(2-\alpha_{n}\right) \eta\right)}{n c \tau \Delta^{\prime} \eta^{p}}$;

$(\mathrm{HHH}) \lim _{\left(\left|x_{1}\right|,\left|x_{2}\right|, \ldots,\left|x_{n}\right|\right) \rightarrow(+\infty,+\infty, \ldots,+\infty)} \sup \frac{F\left(x_{1}, x_{2}, \ldots, x_{n}\right)}{\frac{1}{p} \sum_{i=1}^{n}\left|x_{i}\right|^{p}} \leq 0$.

Then, setting

$$
\begin{aligned}
\Lambda_{2}:= & \left(\frac{n \tau \triangle^{\prime} \eta^{p}}{T(1-2 \gamma) F\left(\Gamma\left(2-\alpha_{1}\right) \eta, \ldots, \Gamma\left(2-\alpha_{n}\right) \eta\right)},\right. \\
& \left.\frac{k d}{c T \max _{\left(x_{1}, x_{2}, \ldots, x_{n}\right) \in K(d)} F\left(x_{1}, x_{2}, \ldots x_{n}\right)}\right),
\end{aligned}
$$

for each compact interval $[a, b] \subseteq \Lambda_{2}$, there exists $\rho>0$ with the following property: for every $\lambda \in] a, b[$, there exists $\delta>0$ such that, for each $\mu \in[0, \delta]$, system (1.4) admits at least three solutions in $X$ whose norms are less than $\rho$.

\section{Acknowledgements}

We authors acknowledge to Prof. Aldo Jonathan Muñoz-Vázquez for a first revision and kind comments on this work. The authors would like to thank the anonymous referees and the handling editor for their careful reading and for relevant remarks/suggestions. 
Availability of data and materials

Not applicable.

\section{Ethics approval and consent to participate}

Not applicable.

\section{Competing interests}

The authors declare that there is no conflict of interests regarding the publication of this manuscript. The authors declare that they have no competing interests.

\section{Consent for publication}

Not applicable.

\section{Authors' contributions}

The authors contributed equally in this article. They have all read and approved the final manuscript.

\section{Author details}

'Department of Mathematics, Faculty of Exact Sciences, Larbi Tebessi University, Tebessa, Algeria. ${ }^{2}$ Department of Mathematics, College of Sciences and Arts, Qassim University, Al-Rass, Kingdom of Saudi Arabia. ${ }^{3}$ Laboratory of Fundamental and Applied Mathematics of Oran (LMFAO), University of Oran 1, Ahmed Benbella, Algeria.

\section{Publisher's Note}

Springer Nature remains neutral with regard to jurisdictional claims in published maps and institutional affiliations.

Received: 29 June 2020 Accepted: 21 July 2020 Published online: 31 July 2020

\section{References}

1. Miller, K.S., Ross, B.: An Introduction to the Fractional Calculus and Fractional Differential Equations. Wiley, New York (1993)

2. Boulaaras, S., Guefaifia, R., Alharbi, A., Cherif, B.: Existence of 3-weak solutions for a new class of an overdetermined system of fractional partial integro-differential equations. Fractals (2020). https://doi.org/10.1142/S0218348X20400368

3. Kamache, F., Guefaifia, R., Boulaaras, S.: Existence of three solutions for perturbed nonlinear fractional p-Laplacian boundary value systems with two control parameters. J. Pseudo-Differ. Oper. Appl. (2020). https://doi.org/10.1007/s11868-020-00354-y

4. Podlubny, I.: Fractional Differential Equations. Academic Press, San Diego (1999)

5. Hilfer, R.: Applications of Fractional Calculus in Physics. World Scientific, Singapore (2000)

6. Kilbas, A.A., Srivastava, H.M., Trujillo, J.J.: Theory and Applications of Fractional Differential Equations. Elsevier, Amsterdam (2006)

7. Abdellaoui, B., Attar, A., Bentifour, R.: On the fractional p-Laplacian equations with weight and general datum. Adv. Nonlinear Anal. 8(1), 144-174 (2019)

8. Wang, P., Ru, Y.: Some existence results of positive solutions for p-Laplacian systems. Bound. Value Probl. 2019, 9 (2019)

9. Gala, S., Liu, Q., Ragusa, M.A.: A new regularity criterion for the nematic liquid crystal fows. Appl. Anal. 91(9), $1741-1747(2012)$

10. Gala, S., Ragusa, M.A.: Logarithmically improved regularity criterion for the Boussinesq equations in Besov spaces with negative indices. Appl. Anal. 95(6), 1271-1279 (2016)

11. Kosmatov, N.: Integral equations and initial value problems for nonlinear differential equations of fractional order. Nonlinear Anal. 70, 2521-2529 (2009)

12. Lakshmikantham, V. Vatsala, A.S. Basic theory of fractional differential equations. Nonlinear Anal. 6, 2677-2682 (2008)

13. Wang, J., Zhou, Y.: A class of fractional evolution equations and optimal controls. Nonlinear Anal., Real World Appl. 12 262-272 (2011)

14. Wei, Z., Dong, W., Che, J.: Periodic boundary value problems for fractional differential equations involving a Riemann-Liouville fractional derivative. Nonlinear Anal. 73, 3232-3238 (2010)

15. Zhang, S.: Positive solutions to singular boundary value problem for nonlinear fractional differential equation. Comput. Math. Appl. 59, 1300-1309 (2010)

16. Fares, K., Rafik, G., Salah, B., Asma, A.: Existence of weak solutions for a new class of fractional p-Laplacian boundary value systems. Mathematics 8, 475 (2020)

17. Mawhin, J., Willem, M.: Critical Point Theory and Hamiltonian Systems. Springer, New York (1989)

18. Rabinowitz, P.H.: Minimax Methods in Critical Point Theory with Applications to Differential Equations. CBMS, vol. 65 Am. Math. Soc. Providence (1986)

19. Willem, M.: Minimax Theorems. Birkhauser, Basel (1996)

20. Mohseni Kolagar, S., Afrouzi, G.A., Hadjian, A.: Existence of three solutions for a class of fractional boundary value systems. Int. J. Nonlinear Anal. Appl. 7, 351-362 (2016)

21. Zhao, Y., Chen, H., Qin, B.: Multiple solutions for a coupled system of nonlinear fractional differential equations via variational methods. Appl. Math. Comput. 257, 417-427 (2015)

22. Zhao, Y., Chen, H., Zhang, Q.: Infinitely many solutions for fractional differential system via variational method. J. Appl. Math. Comput. 50, 589-609 (2016)

23. Ricceri, B.: A three critical points theorem revisited. Nonlinear Anal. 70, 3084-3089 (2009)

24. Chen, T., Liu, W.: Solvability of fractional boundary value problem with $p$-Laplacian via critical point theory. Bound. Value Probl. 2016, 1 (2016) 
25. Li, D., Chen, F., An, Y.: Existence of solutions for fractional differential equation with $p$-Laplacian through variational method. J. Appl. Anal. Comput. 8, 1778-1795 (2018)

26. Bonanno, G., Chinn, A.: Existence of three solutions for a perturbed two-point boundary value problem. Appl. Math. Lett. 23, 807-811 (2010)

27. Candito, P., Agui, G.D.: Three solutions to a perturbed nonlinear discrete Dirichlet problem. J. Math. Anal. Appl. 375, 594-601 (2011)

28. Heidarkhani, S., Henderson, J.: Multiple solutions for a nonlocal perturbed elliptic problem of p-Kirchhoff type. Commun. Appl. Nonlinear Anal. 19, 25-39 (2012)

29. Heidarkhani, S., Henderson, J.: Critical point approaches to quasilinear second order differential equations depending on a parameter. Topol. Methods Nonlinear Anal. 44, 177-197 (2014)

30. Samko, S.G., Kilbas, A.A., Marichev, O.I.: Fractional Integral and Derivatives: Theory and Applications. Gordon and Breach, Longhorne (1993)

31. Jiao, F., Zhou, Y.: Existence results for fractional boundary value problem via critical point theory. Int. J. Bifurc. Chaos Appl. Sci. Eng. 22, 1250086 (2012)

32. Jiao, F., Zhou, Y.: Existence of solutions for a class of fractional boundary value problems via critical point theory. Comput. Math. Appl. 62, 1181-1199 (2011)

\section{Submit your manuscript to a SpringerOpen ${ }^{\circ}$ journal and benefit from:}

- Convenient online submission

- Rigorous peer review

- Open access: articles freely available online

- High visibility within the field

- Retaining the copyright to your article

Submit your next manuscript at $\gg$ springeropen.com 